\title{
Modeling spectra of WR+O colliding wind systems
}

\author{
Grant M. Hill ${ }^{1,2}$, Anthony F.J. Moffat ${ }^{2}$, Nicole St-Louis ${ }^{2}$, \\ and Peter Bartzakos ${ }^{2}$ \\ ${ }^{1}$ McDonald Observatory, University of Texas, USA \\ ${ }^{2}$ Université de Montréal, Canada
}

\section{Introduction}

It is crucial to better understand the winds from massive stars. Not only does their mass loss affect their own evolution but it contributes an amount of energy and momentum to the ISM capable of affecting the chemical and dynamical evolution of their environments. Hot star binaries offer an opportunity to learn more. Colliding winds in such systems might be expected to lead to a bow shock which wraps around the star with the weaker wind. As material flows along the shock, it may give rise to extra line emission. Variations in these emission features may allow the characteristics of the shock region to be deduced thus providing a wealth of information about the winds.

\section{Observations and modeling}

During three contiguous weeks in March and April of 1994 we obtained high S/N, moderate resolution spectra of $7 \mathrm{WR}+\mathrm{O}$ binaries with the UTSO $0.6 \mathrm{~m}$ telescope at Las Campanas. We found evidence for colliding winds in all 4 of the $\mathrm{WC}+\mathrm{O}$ systems (WR 42, WR 48, WR 79, WR 113). The C III $\lambda 5696$ emission lines of all $4 \mathrm{WC}+\mathrm{O}$ systems seem to be made up of two components; an underlying profile, with approximately constant shape that shifts back and forth in wavelength as the two stars orbit each other, and some extra emission, the shape and position of which vary in a different way with the orbital period.

Our working hypothesis is that the extra emission arises in the wind-wind interaction region. We assume that in the absence of the excess emission, the underlying $\mathrm{C}$ III $\lambda 5696$ profile has the flat-topped shape that is normal for single WC7 stars, allowing us to subtract the underlying profile. Lührs (1997) has derived equations to describe the line emission from material flowing along a cone enveloping the $\mathrm{O}$ star. The model is purely geometrical, and assumes optically thin line emission. Fitting profiles of the form described by Lührs' equations provides estimates of the streaming velocity of material in the cone, $v_{\text {strm }}$, the opening half-angle of the cone $\theta$, and its thickness $\Delta \theta$, the orbital inclination $i$, and the Coriolis deflection angle $\delta \phi$ of the axis of the cone with respect to the line joining the stars. Our approach is slightly different from Lührs, who starts with measurements of the excess emission double peaks. Depending on resolution, wind speed and shock cone geometry, the double peaks may not always be easily measurable. Lührs' equations imply that FWHM and radial velocity of the excess emission should vary like: 

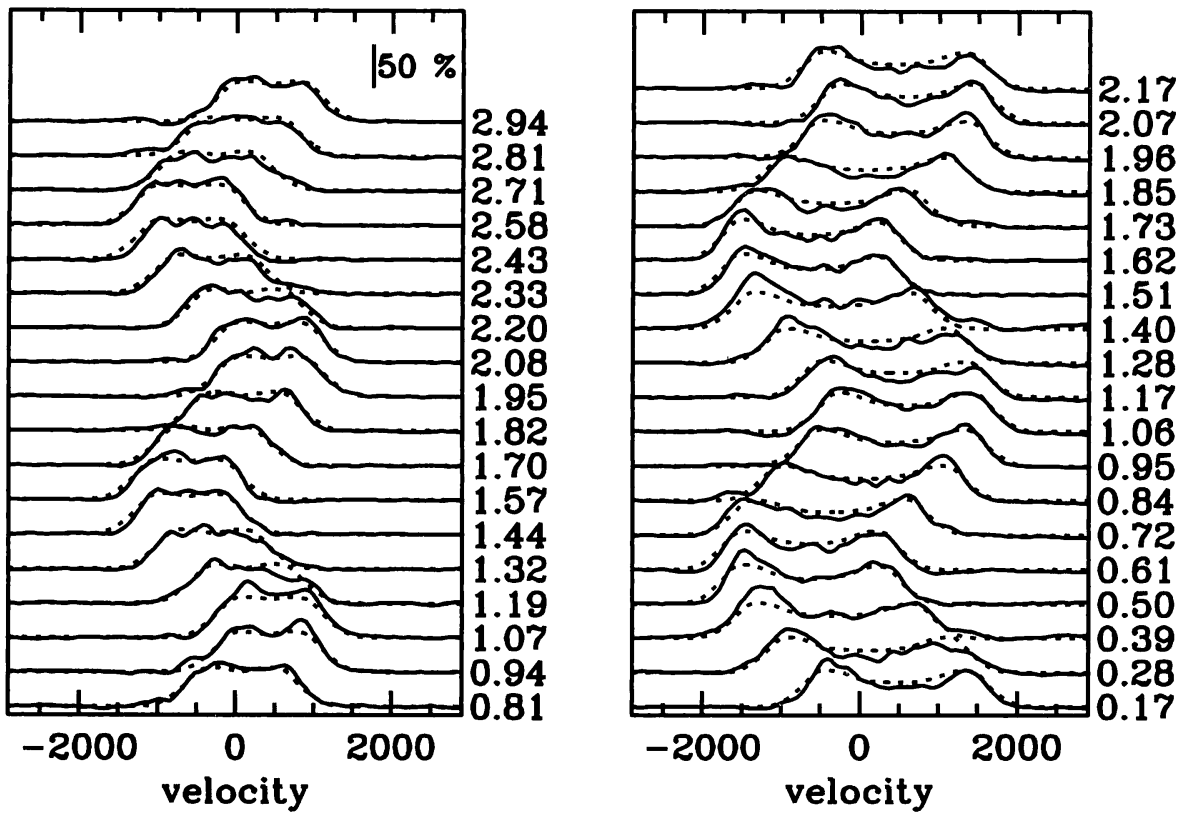

Figure 1. Fit of synthetic profiles (dashed lines) to the WR 42 (left) and WR 79 (right) C III $\lambda 5696$ excess emission.

$$
\begin{gathered}
\text { FWHM (excess) }=\mathrm{C}_{1}+2 v_{\mathrm{str}} \sin \theta \sqrt{1-\sin ^{2} i \cos ^{2}(\phi-\delta \phi)} \\
\mathrm{RV}(\text { excess })=\mathrm{C}_{2}+v_{\mathrm{str}} \cos \theta \sin i \cos (\phi-\delta \phi)
\end{gathered}
$$

where $\mathrm{C}_{1}$ and $\mathrm{C}_{2}$ are simple constants. We have used the results of fitting curves of these forms to our FWHM and radial velocity measurements as initial values for the detailed profile fitting of the excess emission. Figure 1 shows our detailed fits to the observed excess emission and Table 1 presents the derived parameters.

Table 1. Fitting parameters for excess emission

\begin{tabular}{ccc} 
& WR 42 & WR 79 \\
\hline$v_{\text {strm }}$ & $1553 \pm 506 \mathrm{~km} \mathrm{~s}^{-1}$ & $1643 \pm 33 \mathrm{~km} \mathrm{~s}^{-1}$ \\
$\theta$ & $23.2^{\circ} \pm 7.3^{\circ}$ & $46.0^{\circ} \pm 1.3^{\circ}$ \\
$\Delta \theta$ & $6.7^{\circ} \pm 15.4^{\circ}$ & $2.2^{\circ} \pm 2.1^{\circ}$ \\
$i$ & $23.4^{\circ} \pm 6.4^{\circ}$ & $32.8^{\circ} \pm 1.7^{\circ}$ \\
$\delta \phi$ & $3.9^{\circ} \pm 3.0^{\circ}$ & $24.5^{\circ} \pm 1.2^{\circ}$ \\
\hline
\end{tabular}

\section{References}

Lührs, S. 1997, PASP 109, 504 